\title{
Apéndice legal
}

\section{Legal appendix}

\author{
M. Iraburu
}

\section{INTRODUCCIÓN}

Quizá alguien podría extrañarse de la presencia de este apéndice legal en un monográfico de bioética, pero tiene una razón de ser.

La Bioética se ocupa del análisis ético de las situaciones concretas relacionadas con la salud de las personas. Brinda un método que permite reflexionar no sólo en base a datos objetivos sino también a la luz de los valores implicados. Permite sustentar el diálogo racional y la búsqueda de soluciones prudentes ante los conflictos, evitando las respuestas predeterminadas.

Por otra parte, el pluralismo moral y cultural de nuestra sociedad plantea el reto de armonizar diferentes concepciones de vida, de forma que sean respetuosas con los valores y principios constitucionalmente reconocidos y con el núcleo esencial de los derechos fundamentales. Una sociedad pluralista, civil y laica intentará proteger los valores compartidos por la mayoría a través de su ordenamiento jurídico, estableciendo un marco legal de obligado cumplimiento para todos, a partir del cual cada ciudadano podrá desarrollar su propio proyecto vital. Esto, no cabe duda, impone ciertos límites a la libertad individual, pero, por otro lado, la protege de la injerencia de los demás y de que los poderosos impongan "sus verdades".

Las normas legales pueden parecernos injustas, inadecuadas, abusivas, desajustadas a la realidad..., e incluso serlo. Los ciudadanos, respetando las reglas de juego, podremos -y en ocasiones deberíamosluchar para cambiarlas. De hecho, desde un punto de vista ético, el progreso de la sociedad civil vendrá dado, en gran medida, por su capacidad para abrirse a nuevas perspectivas y mantener una constante confrontación racional entre lo viejo y lo nuevo. El libre intercambio de argumentos permitirá, con el tiempo, que las distintas posturas se hagan más permeables, logrando así ampliar el espacio de los valores compartidos y de este modo cimentar la paz social huyendo de la mera imposición.

Por último, y volviendo al ámbito sanitario, el deber ético y jurídico que emana del respeto a la autonomía de las personas ha venido a revolucionar la relación clínica, enriqueciéndola, pero también volviéndola más compleja. Ojalá que los profesionales de la salud no vean el ordenamiento jurídico como algo que les acosa, que les complica la vida, sino como lo que es, un ordenamiento de la sociedad que, aun siendo siempre mejorable, resulta impres-
Médico internista. Coordinadora de la Historia Clínica Informatizada en el Hospital Virgen del Camino. Pamplona.

\section{Correspondencia:}

E-mail: mirabure@cfnavarra.es 
cindible para garantizar las libertades individuales de todos los ciudadanos.

\section{OBJETIVO}

El objetivo de este apartado es facilitar al lector la consulta de las leyes relacionadas con los temas tratados en esta obra.

Por razones de espacio y de rango, he incluido aquí únicamente las leyes estatales más representativas. Todas ellas van precedidas de un breve comentario y, según el tipo de ley, figurará el texto íntegro o sólo los artículos referidos a las cuestiones que nos ocupan.

Por otra parte, dado que el ámbito natural de la revista es Navarra, aparecen las direcciones de Internet para acceder a las leyes navarras más relevantes. Ni que decir tiene que existen leyes homólogas en prácticamente todas las autonomías y que su búsqueda en Internet no presenta tampoco ninguna dificultad.

\section{Leyes estatales}

- Ley 14/1986, de 25 de abril, General de Sanidad. - artículos 9 y 10 (algunos puntos ya derogados). http://noticias.juridicas.com/base_datos/Admin/1141986.html (Visitada el 27/11/2006)

- Ley 41/2002, de 14 de noviembre, básica reguladora de la autonomía del paciente y de derechos y obligaciones en materia de información y documentación clínica. http://noticias.juridicas.com/base_datos /Admin/141-2002.html (Visitada el 27/11/2006)

- Ley Orgánica 15/1999, de 13 de diciembre, de Protección de Datos de Carácter Personal. http://noticias.juridicas.com/ base_datos/Admin/lo15-1999.html (Visitada el 27/11/2006)

- Ley 16/2003, de 28 de mayo, de cohesión y calidad del Sistema Nacional de Salud http://noticias.juridicas.com/base_datos /Admin/l16-2003.html (Visitada el $27 / 11 / 2006)$

\section{Leyes forales navarras}

- Ley Foral 11/2002, de 6 de mayo, sobre los derechos del paciente a las voluntades anticipadas, a la información y a la documentación clínica. http://www.todalaley.com/mostrarLey743p1tn.htm (Visitada el 27/11/2006)

- Ley Foral 29/2003, de 4 de abril, por la que se modifica parcialmente la Ley Foral $11 / 2002$ http://noticias.juridicas.com/base_datos /CCAA/na-l11-2002.html (Visitada el $27 / 11 / 2006)$

\section{Ley 14/1986, de 25 de abril, General de Sanidad}

http://noticias.juridicas.com/base_datos/ Admin/l14-1986.html

(Visitada el 27/11/2006)

Esta ley, por primera vez en España, reconoció el derecho del paciente a autorizar o a rechazar las actuaciones propuestas por los profesionales, tras haber sido debidamente informado. Reguló el "Consentimiento informado" y otros derechos del usuario fundamentalmente en sus artículos 9, 10 y 61. Posteriormente la Ley Básica 41/2002 amplió éstos, derogando algunos puntos del artículo 10 y el artículo 61 en su totalidad.

\section{Artículo Nueve}

Los poderes públicos deberán informar a los usuarios de los servicios del sistema sanitario público, o vinculados a él, de sus derechos y deberes.

\section{Artículo Diez}

Todos tienen los siguientes derechos con respecto a las distintas administraciones públicas sanitarias:

1. Al respeto a su personalidad, dignidad humana e intimidad, sin que pueda ser discriminado por razones de raza, de tipo social, de sexo, moral, económico, ideológico, político o sindical.

2. A la información sobre los servicios sanitarios a que puede acceder y sobre los requisitos necesarios para su uso.

3. A la confidencialidad de toda la información relacionada con su proceso y con su estancia en instituciones sanitarias públicas y privadas que colaboren con el sistema público.

4. A ser advertido de si los procedimientos de pronóstico, diagnóstico y tera- 
péuticos que se le apliquen pueden ser utilizados en función de un proyecto docente o de investigación, que, en ningún caso, podrá comportar peligro adicional para su salud. En todo caso será imprescindible la previa autorización, y por escrito, del paciente y la aceptación por parte del médico y de la dirección del correspondiente centro sanitario.

5. - Derogado por la Ley $41 / 2002$

6. - Derogado por la Ley $41 / 2002$

7. A que se le asigne un médico, cuyo nombre se le dará a conocer, que será su interlocutor principal con el equipo asistencial. En caso de ausencia, otro facultativo del equipo asumirá tal responsabilidad.

8. - Derogado por la Ley $41 / 2002$

9. - Derogado por la Ley $41 / 2002$

10. A participar, a través de las instituciones comunitarias, en las actividades sanitarias, en los términos establecidos en esta Ley y en las disposiciones que la desarrollen.

\section{1. - Derogado por la Ley $41 / 2002$}

12. A utilizar las vías de reclamación y de propuesta de sugerencias en los plazos previstos. En uno u otro caso deberá recibir respuesta por escrito en los plazos que reglamentariamente se establezcan.

13. A elegir el médico y los demás sanitarios titulados de acuerdo con las condiciones contempladas en esta Ley, en las disposiciones que se dicten para su desarrollo y en las que regule el trabajo sanitario en los centros de Salud.

14. A obtener los medicamentos y productos sanitarios que se consideren necesarios para promover, conservar o restablecer su salud, en los términos que reglamentariamente se establezcan por la Administración del Estado.

15. Respetando el peculiar régimen económico de cada servicio sanitario, los derechos contemplados en los apartados $1,3,4,5,6,7,9$ y 11 de este artículo serán ejercidos también con respecto a los servicios sanitarios privados.
Ley 41/2002, de 14 de noviembre, básica reguladora de la autonomía del paciente y de derechos y obligaciones en materia de información y documentación clínica.

http://noticias.juridicas.com/base_datos/ Admin/141-2002.html

(Visitada el 27/11/2006)

Esta Ley ha supuesto un hito para el mundo sanitario.

Mejora, respecto a la Ley General de Sanidad de 1986, los aspectos relacionados con la información y el consentimiento, regula aspectos de gran relevancia sobre la Historia Clínica y la documentación, introduce importantes novedades en torno al paciente menor de edad y otorga validez legal a las instrucciones previas como una expresión más de la autonomía personal.

Fue una Ley ampliamente debatida y consensuada y, aunque contiene aspectos poco precisos o incluso polémicos, hay que reconocerle el mérito de haber clarificado enormemente los derechos y deberes de todos los implicados en la relación clínica: usuarios, profesionales e institución. Está además escrita de manera clara y asequible, y tiene un preámbulo que sitúa muy bien el momento histórico de su génesis.

La incluyo íntegra porque considero que todos los profesionales sanitarios debieran leerla y consultarla con frecuencia.

Es una Ley que hay que tener a mano. Al fin y al cabo nos dice qué podemos y qué no podemos hacer en nuestra tarea profesional.

\section{JUAN CARLOS I \\ REY DE ESPAÑA}

A todos los que la presente vieren y entendieren.

Sabed: Que las Cortes Generales han aprobado y Yo vengo en sancionar la siguiente Ley.

\section{EXPOSICIÓN DE MOTIVOS}

La importancia que tienen los derechos de los pacientes como eje básico de las relaciones clínico-asistenciales se pone de manifiesto al constatar el interés que han demostrado por los mismos casi todas las organizaciones internacionales con com- 
petencia en la materia. Ya desde el fin de la Segunda Guerra Mundial, organizaciones como Naciones Unidas, UNESCO o la Organización Mundial de la Salud, o, más recientemente, la Unión Europea o el Consejo de Europa, entre muchas otras, han impulsado declaraciones o, en algún caso, han promulgado normas jurídicas sobre aspectos genéricos o específicos relacionados con esta cuestión. En este sentido, es necesario mencionar la trascendencia de la Declaración Universal de Derechos Humanos, del año 1948, que ha sido el punto de referencia obligado para todos los textos constitucionales promulgados posteriormente o, en el ámbito más estrictamente sanitario, la Declaración sobre la promoción de los derechos de los pacientes en Europa, promovida el año 1994 por la Oficina Regional para Europa de la Organización Mundial de la Salud, aparte de múltiples declaraciones internacionales de mayor o menor alcance e influencia que se han referido a dichas cuestiones.

Últimamente, cabe subrayar la relevancia especial del Convenio del Consejo de Europa para la protección de los derechos humanos y la dignidad del ser humano respecto de las aplicaciones de la biología y la medicina (Convenio sobre los derechos del hombre y la biomedicina), suscrito el día 4 de abril de 1997, el cual ha entrado en vigor en el Reino de España el 1 de enero de 2000. Dicho Convenio es una iniciativa capital: en efecto, a diferencia de las distintas declaraciones internacionales que lo han precedido, es el primer instrumento internacional con carácter jurídico vinculante para los países que lo suscriben. Su especial valía reside en el hecho de que establece un marco común para la protección de los derechos humanos y la dignidad humana en la aplicación de la biología y la medicina. El Convenio trata explícitamente, con detenimiento y extensión, sobre la necesidad de reconocer los derechos de los pacientes, entre los cuales resaltan el derecho a la información, el consentimiento informado y la intimidad de la información relativa a la salud de las personas, persiguiendo el alcance de una armonización de las legislaciones de los diversos países en estas materias; en este sentido, es absolutamente conveniente tener en cuenta el Convenio en el momento de abordar el reto de regular cuestiones tan importantes.

Es preciso decir, sin embargo, que la regulación del derecho a la protección de la salud, recogido por el artículo 43 de la Constitución de 1978, desde el punto de vista de las cuestiones más estrechamente vinculadas a la condición de sujetos de derechos de las personas usuarias de los servicios sanitarios, es decir, la plasmación de los derechos relativos a la información clínica y la autonomía individual de los pacientes en lo relativo a su salud, ha sido objeto de una regulación básica en el ámbito del Estado, a través de la Ley 14/1986, de 25 de abril, General de Sanidad.

De otra parte, esta Ley, a pesar de que fija básicamente su atención en el establecimiento y ordenación del sistema sanitario desde un punto de vista organizativo, dedica a esta cuestión diversas previsiones, entre las que destaca la voluntad de humanización de los servicios sanitarios. Así mantiene el máximo respeto a la dignidad de la persona y a la libertad individual, de un lado, y, del otro, declara que la organización sanitaria debe permitir garantizar la salud como derecho inalienable de la población mediante la estructura del Sistema Nacional de Salud, que debe asegurarse en condiciones de escrupuloso respeto a la intimidad personal y a la libertad individual del usuario, garantizando la confidencialidad de la información relacionada con los servicios sanitarios que se prestan y sin ningún tipo de discriminación.

A partir de dichas premisas, la presente Ley completa las previsiones que la Ley General de Sanidad enunció como principios generales. En este sentido, refuerza y da un trato especial al derecho a la autonomía del paciente. En particular, merece mención especial la regulación sobre instrucciones previas que contempla, de acuerdo con el criterio establecido en el Convenio de Oviedo, los deseos del paciente expresados con anterioridad dentro del ámbito del consentimiento informado. Asimismo, la Ley trata con profundidad todo lo referente a la documentación clínica generada en los centros asistenciales, subrayando especialmente la considera- 
ción y la concreción de los derechos de los usuarios en este aspecto.

En septiembre de 1997, en desarrollo de un convenio de colaboración entre el Consejo General del Poder Judicial y el Ministerio de Sanidad y Consumo, tuvo lugar un seminario conjunto sobre información y documentación clínica, en el que se debatieron los principales aspectos normativos y judiciales en la materia. Al mismo tiempo, se constituyó un grupo de expertos a quienes se encargó la elaboración de unas directrices para el desarrollo futuro de este tema. Este grupo suscribió un dictamen el 26 de noviembre de 1997, que ha sido tenido en cuenta en la elaboración de los principios fundamentales de esta Ley.

La atención que a estas materias otorgó en su día la Ley General de Sanidad supuso un notable avance como reflejan, entre otros, sus artículos 9, 10 y 61. Sin embargo, el derecho a la información, como derecho del ciudadano cuando demanda la atención sanitaria, ha sido objeto en los últimos años de diversas matizaciones y ampliaciones por Leyes y disposiciones de distinto tipo y rango, que ponen de manifiesto la necesidad de una reforma y actualización de la normativa contenida en la Ley General de Sanidad. Así, la Ley Orgánica 15/1999, de 13 de diciembre, de Protección de Datos de Carácter Personal, califica a los datos relativos a la salud de los ciudadanos como datos especialmente protegidos, estableciendo un régimen singularmente riguroso para su obtención, custodia y eventual cesión. Esta defensa de la confidencialidad había sido ya defendida por la Directiva comunitaria 95/46, de 24 de octubre, en la que, además de reafirmarse la defensa de los derechos y libertades de los ciudadanos europeos, en especial de su intimidad relativa a la información relacionada con su salud, se apunta la presencia de otros intereses generales como los estudios epidemiológicos, las situaciones de riesgo grave para la salud de la colectividad, la investigación y los ensayos clínicos que, cuando estén incluidos en normas de rango de Ley, pueden justificar una excepción motivada a los derechos del paciente. Se manifiesta así una concepción comuni- taria del derecho a la salud, en la que, junto al interés singular de cada individuo, como destinatario por excelencia de la información relativa a la salud, aparecen también otros agentes y bienes jurídicos referidos a la salud pública, que deben ser considerados, con la relevancia necesaria, en una sociedad democrática avanzada. En esta línea, el Consejo de Europa, en su Recomendación de 13 de febrero de 1997, relativa a la protección de los datos médicos, después de afirmar que deben recogerse y procesarse con el consentimiento del afectado, indica que la información puede restringirse si así lo dispone una Ley y constituye una medida necesaria por razones de interés general.

Todas estas circunstancias aconsejan una adaptación de la Ley General de Sanidad con el objetivo de aclarar la situación jurídica y los derechos y obligaciones de los profesionales sanitarios, de los ciudadanos y de las instituciones sanitarias. Se trata de ofrecer en el terreno de la información y la documentación clínicas las mismas garantías a todos los ciudadanos del Estado, fortaleciendo con ello el derecho a la protección de la salud que reconoce la Constitución.

\section{CAPÍTULO I \\ Principios generales}

\section{Artículo 1. Ámbito de aplicación}

La presente Ley tiene por objeto la regulación de los derechos y obligaciones de los pacientes, usuarios y profesionales, así como de los centros y servicios sanitarios, públicos y privados, en materia de autonomía del paciente y de información y documentación clínica.

\section{Artículo 2. Principios básicos}

1. La dignidad de la persona humana, el respeto a la autonomía de su voluntad y a su intimidad orientarán toda la actividad encaminada a obtener, utilizar, archivar, custodiar y transmitir la información y la documentación clínica.

2. Toda actuación en el ámbito de la sanidad requiere, con carácter general, el previo consentimiento de los pacientes o usuarios. El consentimiento, que debe obtenerse después de que el paciente reciba una información adecuada, se hará por 
escrito en los supuestos previstos en la Ley.

3. El paciente o usuario tiene derecho a decidir libremente, después de recibir la información adecuada, entre las opciones clínicas disponibles.

4. Todo paciente o usuario tiene derecho a negarse al tratamiento, excepto en los casos determinados en la Ley. Su negativa al tratamiento constará por escrito.

5. Los pacientes o usuarios tienen el deber de facilitar los datos sobre su estado físico o sobre su salud de manera leal y verdadera, así como el de colaborar en su obtención, especialmente cuando sean necesarios por razones de interés público o con motivo de la asistencia sanitaria.

6. Todo profesional que interviene en la actividad asistencial está obligado no sólo a la correcta prestación de sus técnicas, sino al cumplimiento de los deberes de información y de documentación clínica, y al respeto de las decisiones adoptadas libre y voluntariamente por el paciente.

7. La persona que elabore o tenga acceso a la información y la documentación clínica está obligada a guardar la reserva debida.

\section{CAPÍTULO II \\ El derecho de información sanitaria}

\section{Artículo 4. Derecho a la información asis- tencial}

1. Los pacientes tienen derecho a conocer, con motivo de cualquier actuación en el ámbito de su salud, toda la información disponible sobre la misma, salvando los supuestos exceptuados por la Ley. Además, toda persona tiene derecho a que se respete su voluntad de no ser informada. La información, que como regla general se proporcionará verbalmente dejando constancia en la historia clínica, comprende, como mínimo, la finalidad y la naturaleza de cada intervención, sus riesgos y sus consecuencias.

2. La información clínica forma parte de todas las actuaciones asistenciales, será verdadera, se comunicará al paciente de forma comprensible y adecuada a sus necesidades y le ayudará a tomar decisio- nes de acuerdo con su propia y libre voluntad.

3. El médico responsable del paciente le garantiza el cumplimiento de su derecho a la información. Los profesionales que le atiendan durante el proceso asistencial o le apliquen una técnica o un procedimiento concreto también serán responsables de informarle.

\section{Artículo 5. Titular del derecho a la infor- mación asistencial}

1. El titular del derecho a la información es el paciente. También serán informadas las personas vinculadas a él, por razones familiares o de hecho, en la medida que el paciente lo permita de manera expresa o tácita.

2. El paciente será informado, incluso en caso de incapacidad, de modo adecuado a sus posibilidades de comprensión, cumpliendo con el deber de informar también a su representante legal.

3. Cuando el paciente, según el criterio del médico que le asiste, carezca de capacidad para entender la información a causa de su estado físico o psíquico, la información se pondrá en conocimiento de las personas vinculadas a él por razones familiares o de hecho.

4. El derecho a la información sanitaria de los pacientes puede limitarse por la existencia acreditada de un estado de necesidad terapéutica. Se entenderá por necesidad terapéutica la facultad del médico para actuar profesionalmente sin informar antes al paciente, cuando por razones objetivas el conocimiento de su propia situación pueda perjudicar su salud de manera grave. Llegado este caso, el médico dejará constancia razonada de las circunstancias en la historia clínica y comunicará su decisión a las personas vinculadas al paciente por razones familiares o de hecho.

\section{Artículo 6. Derecho a la información epi- demiológica}

Los ciudadanos tienen derecho a conocer los problemas sanitarios de la colectividad cuando impliquen un riesgo para la salud pública o para su salud individual, y el derecho a que esta información se difunda en términos verdaderos, comprensibles 
y adecuados para la protección de la salud, de acuerdo con lo establecido por la Ley.

\section{CAPÍTULO III \\ Derecho a la intimidad}

\section{Artículo 7. El derecho a la intimidad}

1. Toda persona tiene derecho a que se respete el carácter confidencial de los datos referentes a su salud, y a que nadie pueda acceder a ellos sin previa autorización amparada por la Ley.

2. Los centros sanitarios adoptarán las medidas oportunas para garantizar los derechos a que se refiere el apartado anterior, y elaborarán, cuando proceda, las normas y los procedimientos protocolizados que garanticen el acceso legal a los datos de los pacientes.

\section{CAPÍTULO IV}

\section{El respeto de la autonomía del paciente}

\section{Artículo 8. Consentimiento informado}

1. Toda actuación en el ámbito de la salud de un paciente necesita el consentimiento libre y voluntario del afectado, una vez que, recibida la información prevista en el artículo 4, haya valorado las opciones propias del caso.

2. El consentimiento será verbal por regla general. Sin embargo, se prestará por escrito en los casos siguientes: intervención quirúrgica, procedimientos diagnósticos y terapéuticos invasores y, en general, aplicación de procedimientos que suponen riesgos o inconvenientes de notoria y previsible repercusión negativa sobre la salud del paciente.

3 . El consentimiento escrito del paciente será necesario para cada una de las actuaciones especificadas en el punto anterior de este artículo, dejando a salvo la posibilidad de incorporar anejos y otros datos de carácter general, y tendrá información suficiente sobre el procedimiento de aplicación y sobre sus riesgos.

4. Todo paciente o usuario tiene derecho a ser advertido sobre la posibilidad de utilizar los procedimientos de pronóstico, diagnóstico y terapéuticos que se le apliquen en un proyecto docente o de investi- gación, que en ningún caso podrá comportar riesgo adicional para su salud.

5. El paciente puede revocar libremente por escrito su consentimiento en cualquier momento.

Artículo 9. Límites del consentimiento informado y consentimiento por representación

1. La renuncia del paciente a recibir información está limitada por el interés de la salud del propio paciente, de terceros, de la colectividad y por las exigencias terapéuticas del caso. Cuando el paciente manifieste expresamente su deseo de no ser informado, se respetará su voluntad haciendo constar su renuncia documentalmente, sin perjuicio de la obtención de su consentimiento previo para la intervención.

2. Los facultativos podrán llevar a cabo las intervenciones clínicas indispensables en favor de la salud del paciente, sin necesidad de contar con su consentimiento, en los siguientes casos:

a) Cuando existe riesgo para la salud pública a causa de razones sanitarias establecidas por la Ley. En todo caso, una vez adoptadas las medidas pertinentes, de conformidad con lo establecido en la Ley Orgánica 3/1986, se comunicarán a la autoridad judicial en el plazo máximo de 24 horas siempre que dispongan el internamiento obligatorio de personas.

b) Cuando existe riesgo inmediato grave para la integridad física o psíquica del enfermo y no es posible conseguir su autorización, consultando, cuando las circunstancias lo permitan, a sus familiares o a las personas vinculadas de hecho a él.

3. Se otorgará el consentimiento por representación en los siguientes supuestos:

a) Cuando el paciente no sea capaz de tomar decisiones, a criterio del médico responsable de la asistencia, o su estado físico o psíquico no le permita hacerse cargo de su situación. Si el paciente carece de representante legal, el consentimiento lo prestarán las personas vinculadas a él por razones familiares o de hecho.

b) Cuando el paciente esté incapacitado legalmente. 
c) Cuando el paciente menor de edad no sea capaz intelectual ni emocionalmente de comprender el alcance de la intervención. En este caso, el consentimiento lo dará el representante legal del menor después de haber escuchado su opinión si tiene doce años cumplidos. Cuando se trate de menores no incapaces ni incapacitados, pero emancipados o con dieciséis años cumplidos, no cabe prestar el consentimiento por representación. Sin embargo, en caso de actuación de grave riesgo, según el criterio del facultativo, los padres serán informados y su opinión será tenida en cuenta para la toma de la decisión correspondiente.

4. La interrupción voluntaria del embarazo, la práctica de ensayos clínicos y la práctica de técnicas de reproducción humana asistida se rigen por lo establecido con carácter general sobre la mayoría de edad y por las disposiciones especiales de aplicación.

5. La prestación del consentimiento por representación será adecuada a las circunstancias y proporcionada a las necesidades que haya que atender, siempre en favor del paciente y con respeto a su dignidad personal. El paciente participará en la medida de lo posible en la toma de decisiones a lo largo del proceso sanitario.

Artículo 10. Condiciones de la información y consentimiento por escrito

1. El facultativo proporcionará al paciente, antes de recabar su consentimiento escrito, la información básica siguiente:

a) Las consecuencias relevantes o de importancia que la intervención origina con seguridad.

b) Los riesgos relacionados con las circunstancias personales o profesionales del paciente.

c) Los riesgos probables en condiciones normales, conforme a la experiencia y al estado de la ciencia o directamente relacionados con el tipo de intervención.

d) Las contraindicaciones.

2. El médico responsable deberá ponderar en cada caso que cuanto más dudoso sea el resultado de una intervención más necesario resulta el previo consentimiento por escrito del paciente.

\section{Artículo 11. Instrucciones previas}

1. Por el documento de instrucciones previas, una persona mayor de edad, capaz y libre, manifiesta anticipadamente su voluntad, con objeto de que ésta se cumpla en el momento en que llegue a situaciones en cuyas circunstancias no sea capaz de expresarlos personalmente, sobre los cuidados y el tratamiento de su salud o, una vez llegado el fallecimiento, sobre el destino de su cuerpo o de los órganos del mismo. El otorgante del documento puede designar, además, un representante para que, llegado el caso, sirva como interlocutor suyo con el médico o el equipo sanitario para procurar el cumplimiento de las instrucciones previas.

2. Cada servicio de salud regulará el procedimiento adecuado para que, llegado el caso, se garantice el cumplimiento de las instrucciones previas de cada persona, que deberán constar siempre por escrito.

3. No serán aplicadas las instrucciones previas contrarias al ordenamiento jurídico, a la "lex artis", ni las que no se correspondan con el supuesto de hecho que el interesado haya previsto en el momento de manifestarlas. En la historia clínica del paciente quedará constancia razonada de las anotaciones relacionadas con estas previsiones.

4. Las instrucciones previas podrán revocarse libremente en cualquier momento dejando constancia por escrito.

5. Con el fin de asegurar la eficacia en todo el territorio nacional de las instrucciones previas manifestadas por los pacientes y formalizadas de acuerdo con lo dispuesto en la legislación de las respectivas Comunidades Autónomas, se creará en el Ministerio de Sanidad y Consumo el Registro nacional de instrucciones previas que se regirá por las normas que reglamentariamente se determinen, previo acuerdo del Consejo Interterritorial del Sistema Nacional de Salud.

\section{Artículo 12. Información en el Sistema Nacional de Salud}

1. Además de los derechos reconocidos en los artículos anteriores, los pacientes y 
los usuarios del Sistema Nacional de Salud tendrán derecho a recibir información sobre los servicios y unidades asistenciales disponibles, su calidad y los requisitos de acceso a ellos.

2. Los servicios de salud dispondrán en los centros y servicios sanitarios de una guía o carta de los servicios en la que se especifiquen los derechos y obligaciones de los usuarios, las prestaciones disponibles, las características asistenciales del centro o del servicio, y sus dotaciones de personal, instalaciones y medios técnicos. Se facilitará a todos los usuarios información sobre las guías de participación y sobre sugerencias y reclamaciones.

3. Cada servicio de salud regulará los procedimientos y los sistemas para garantizar el efectivo cumplimiento de las previsiones de este artículo.

\section{Artículo 13. Derecho a la información para la elección de médico y de centro}

Los usuarios y pacientes del Sistema Nacional de Salud, tanto en la atención primaria como en la especializada, tendrán derecho a la información previa correspondiente para elegir médico, e igualmente centro, con arreglo a los términos y condiciones que establezcan los servicios de salud competentes.

\section{CAPÍTULO V \\ La historia clínica}

\section{Artículo 15. Contenido de la historia clí- nica de cada paciente}

1. La historia clínica incorporará la información que se considere trascendental para el conocimiento veraz y actualizado del estado de salud del paciente. Todo paciente o usuario tiene derecho a que quede constancia, por escrito o en el soporte técnico más adecuado, de la información obtenida en todos sus procesos asistenciales, realizados por el servicio de salud tanto en el ámbito de atención primaria como de atención especializada.

2. La historia clínica tendrá como fin principal facilitar la asistencia sanitaria, dejando constancia de todos aquellos datos que, bajo criterio médico, permitan el conocimiento veraz y actualizado del estado de salud.

\section{Artículo 16. Usos de la historia clínica}

1. La historia clínica es un instrumento destinado fundamentalmente a garantizar una asistencia adecuada al paciente. Los profesionales asistenciales del centro que realizan el diagnóstico o el tratamiento del paciente tienen acceso a la historia clínica de éste como instrumento fundamental para su adecuada asistencia.

2. Cada centro establecerá los métodos que posibiliten en todo momento el acceso a la historia clínica de cada paciente por los profesionales que le asisten.

3. El acceso a la historia clínica con fines judiciales, epidemiológicos, de salud pública, de investigación o de docencia, se rige por lo dispuesto en la Ley Orgánica 15/1999, de Protección de Datos de Carácter Personal, y en la Ley 14/1986, General de Sanidad, y demás normas de aplicación en cada caso. El acceso a la historia clínica con estos fines obliga a preservar los datos de identificación personal del paciente, separados de los de carácter clínico-asistencial, de manera que como regla general quede asegurado el anonimato, salvo que el propio paciente haya dado su consentimiento para no separarlos. Se exceptúan los supuestos de investigación de la autoridad judicial en los que se considere imprescindible la unificación de los datos identificativos con los clínico-asistenciales, en los cuales se estará a lo que dispongan los jueces y tribunales en el proceso correspondiente. El acceso a los datos y documentos de la historia clínica queda limitado estrictamente a los fines específicos de cada caso.

4. El personal de administración y gestión de los centros sanitarios sólo puede acceder a los datos de la historia clínica relacionados con sus propias funciones.

5. El personal sanitario debidamente acreditado que ejerza funciones de inspección, evaluación, acreditación y planificación, tiene acceso a las historias clínicas en el cumplimiento de sus funciones de comprobación de la calidad de la asistencia, el respeto de los derechos del paciente o cualquier otra obligación del centro en relación con los pacientes y usuarios o la propia Administración sanitaria. 
6. El personal que accede a los datos de la historia clínica en el ejercicio de sus funciones queda sujeto al deber de secreto.

7. Las Comunidades Autónomas regularán el procedimiento para que quede constancia del acceso a la historia clínica y de su uso.

\section{Artículo 18. Derechos de acceso a la his- toria clínica}

1. El paciente tiene el derecho de acceso, con las reservas señaladas en el apartado 3 de este artículo, a la documentación de la historia clínica y a obtener copia de los datos que figuran en ella. Los centros sanitarios regularán el procedimiento que garantice la observancia de estos derechos.

2. El derecho de acceso del paciente a la historia clínica puede ejercerse también por representación debidamente acreditada.

3. El derecho al acceso del paciente a la documentación de la historia clínica no puede ejercitarse en perjuicio del derecho de terceras personas a la confidencialidad de los datos que constan en ella recogidos en interés terapéutico del paciente, ni en perjuicio del derecho de los profesionales participantes en su elaboración, los cuales pueden oponer al derecho de acceso la reserva de sus anotaciones subjetivas.

4. Los centros sanitarios y los facultativos de ejercicio individual sólo facilitarán el acceso a la historia clínica de los pacientes fallecidos a las personas vinculadas a él, por razones familiares o de hecho, salvo que el fallecido lo hubiese prohibido expresamente y así se acredite. En cualquier caso el acceso de un tercero a la historia clínica motivado por un riesgo para su salud se limitará a los datos pertinentes. No se facilitará información que afecte a la intimidad del fallecido ni a las anotaciones subjetivas de los profesionales, ni que perjudique a terceros.

\section{Artículo 19. Derechos relacionados con} la custodia de la historia clínica

El paciente tiene derecho a que los centros sanitarios establezcan un mecanismo de custodia activa y diligente de las historias clínicas. Dicha custodia permitirá la recogida, la integración, la recuperación y la comunicación de la información someti- da al principio de confidencialidad con arreglo a lo establecido por el artículo 16 de la presente Ley.

\section{CAPÍTULO VI}

\section{Informe de alta y otra documentación} clínica

\section{Artículo 20. Informe de alta}

Todo paciente, familiar o persona vinculada a él, en su caso, tendrá el derecho a recibir del centro o servicio sanitario, una vez finalizado el proceso asistencial, un informe de alta con los contenidos mínimos que determina el artículo 3. Las características, requisitos y condiciones de los informes de alta se determinarán reglamentariamente por las Administraciones sanitarias autonómicas.

\section{Artículo 21. El alta del paciente}

1. En caso de no aceptar el tratamiento prescrito, se propondrá al paciente o usuario la firma del alta voluntaria. Si no la firmara, la dirección del centro sanitario, a propuesta del médico responsable, podrá disponer el alta forzosa en las condiciones reguladas por la Ley. El hecho de no aceptar el tratamiento prescrito no dará lugar al alta forzosa cuando existan tratamientos alternativos, aunque tengan carácter paliativo, siempre que los preste el centro sanitario y el paciente acepte recibirlos. Estas circunstancias quedarán debidamente documentadas.

2. En el caso de que el paciente no acepte el alta, la dirección del centro, previa comprobación del informe clínico correspondiente, oirá al paciente y, si persiste en su negativa, lo pondrá en conocimiento del juez para que confirme o revoque la decisión.

\section{Artículo 22. Emisión de certificados médicos}

Todo paciente o usuario tiene derecho a que se le faciliten los certificados acreditativos de su estado de salud. Éstos serán gratuitos cuando así lo establezca una disposición legal o reglamentaria.

\section{Artículo 23. Obligaciones profesionales de información técnica, estadística y administrativa}

Los profesionales sanitarios, además de las obligaciones señaladas en materia 
de información clínica, tienen el deber de cumplimentar los protocolos, registros, informes, estadísticas y demás documentación asistencial o administrativa, que guarden relación con los procesos clínicos en los que intervienen, y los que requieran los centros o servicios de salud competentes y las autoridades sanitarias, comprendidos los relacionados con la investigación médica y la información epidemiológica.

\section{Disposición adicional primera. Carácter de legislación básica}

Esta Ley tiene la condición de básica, de conformidad con lo establecido en el artículo 149.1.1 $1^{\mathrm{a}}$ y $16^{\mathrm{a}}$ de la Constitución.

El Estado y las Comunidades Autónomas adoptarán, en el ámbito de sus respectivas competencias, las medidas necesarias para la efectividad de esta Ley.

\section{Disposición adicional segunda. Aplica- ción supletoria}

Las normas de esta Ley relativas a la información asistencial, la información para el ejercicio de la libertad de elección de médico y de centro, el consentimiento informado del paciente y la documentación clínica, serán de aplicación supletoria en los proyectos de investigación médica, en los procesos de extracción y trasplante de órganos, en los de aplicación de técnicas de reproducción humana asistida y en los que carezcan de regulación especial.

\section{Disposición adicional tercera. Coordina- ción de las historias clínicas}

El Ministerio de Sanidad y Consumo, en coordinación y con la colaboración de las Comunidades Autónomas competentes en la materia, promoverá, con la participación de todos los interesados, la implantación de un sistema de compatibilidad que, atendida la evolución y disponibilidad de los recursos técnicos, y la diversidad de sistemas y tipos de historias clínicas, posibilite su uso por los centros asistenciales de España que atiendan a un mismo paciente, en evitación de que los atendidos en diversos centros se sometan a exploraciones y procedimientos de innecesaria repetición.

\section{Disposición adicional cuarta. Necesida- des asociadas a la discapacidad}

El Estado y las Comunidades Autónomas, dentro del ámbito de sus respectivas competencias, dictarán las disposiciones precisas para garantizar a los pacientes o usuarios con necesidades especiales, asociadas a la discapacidad, los derechos en materia de autonomía, información y documentación clínica regulados en esta Ley.

Disposición adicional quinta. Información y documentación sobre medicamentos y productos sanitarios

La información, la documentación y la publicidad relativas a los medicamentos y productos sanitarios, así como el régimen de las recetas y de las órdenes de prescripción correspondientes, se regularán por su normativa específica, sin perjuicio de la aplicación de las reglas establecidas en esta Ley en cuanto a la prescripción y uso de medicamentos o productos sanitarios durante los procesos asistenciales.

\section{Disposición adicional sexta. Régimen san- cionador}

Las infracciones de lo dispuesto por la presente Ley quedan sometidas al régimen sancionador previsto en el capítulo VI del Título I de la Ley 14/1986, General de Sanidad, sin perjuicio de la responsabilidad civil o penal y de la responsabilidad profesional o estatutaria procedentes en derecho.

\section{Disposición transitoria única. Informe de alta}

El informe de alta se regirá por lo dispuesto en la Orden del Ministerio de Sanidad, de 6 de septiembre de 1984 (RCL 1984, 2254; ApNDL 12303), mientras no se desarrolle legalmente lo dispuesto en el artículo 20 de esta Ley.

Disposición derogatoria única. Derogación general y de preceptos concretos

Quedan derogadas las disposiciones de igual o inferior rango que se opongan a lo dispuesto en la presente Ley y, concretamente, los apartados 5, 6, 8, 9 y 11 del artículo 10, el apartado 4 del artículo 11 y el artículo 61 de la Ley 14/1986, General de Sanidad. 


\section{Disposición final única. Entrada en vigor}

La presente Ley entrará en vigor en el plazo de seis meses a partir del día siguiente al de su publicación en el "Boletín Oficial del Estado".

Ley Orgánica 15/1999, de 13 de diciembre, de protección de datos de carácter personal.

http://noticias.juridicas.com/base_dat os/Admin/lo15-1999.html

(Visitada el 27/11/2006)

Esta Ley regula la protección de cualquier dato que permita identificar a las personas.

Más allá de proteger la intimidad, protege la identidad. Regula el tratamiento de los datos personales en cualquier ámbito, no sólo en el sanitario, y es la causante de la nota al pié de cualquier factura en la que se nos asegura que nuestros datos no serán cedidos a terceros ni utilizados con un fin distinto de aquél para el que fueron recabados.

Por ocuparse de escenarios tan variados como los archivos policiales, los de Hacienda etc., he entresacado aquellos artículos relacionados con el tratamiento de los datos en el entorno clínico.

Al final, figura el índice de otros apartados de la Ley para que el lector conozca de su experiencia

\section{Artículo 1. Objeto}

La presente Ley Orgánica tiene por objeto garantizar y proteger, en lo que concierne al tratamiento de los datos personales, las libertades públicas y los derechos fundamentales de las personas físicas, y especialmente de su honor e intimidad personal y familiar.

\section{Artículo 2. Ámbito de aplicación}

1. La presente Ley Orgánica será de aplicación a los datos de carácter personal registrados en soporte físico, que los haga susceptibles de tratamiento, y a toda modalidad de uso posterior de estos datos por los sectores público y privado

\section{Artículo 3. Definiciones}

A los efectos de la presente Ley Orgánica se entenderá por:

a) Datos de carácter personal: cualquier información concerniente a personas físicas identificadas o identificables.

b) Fichero: todo conjunto organizado de datos de carácter personal, cualquiera que fuere la forma o modalidad de su creación, almacenamiento, organización y acceso.

c) Tratamiento de datos: operaciones y procedimientos técnicos de carácter automatizado o no, que permitan la recogida, grabación, conservación, elaboración, modificación, bloqueo y cancelación, así como las cesiones de datos que resulten de comunicaciones, consultas, interconexiones y transferencias.

d) Responsable del fichero o tratamiento: persona física o jurídica, de naturaleza pública o privada, u órgano administrativo, que decida sobre la finalidad, contenido y uso del tratamiento.

e) Afectado o interesado: persona física titular de los datos que sean objeto del tratamiento a que se refiere el apartado c) del presente artículo.

f) Procedimiento de disociación: todo tratamiento de datos personales de modo que la información que se obtenga no pueda asociarse a persona identificada o identificable.

g) Encargado del tratamiento: la persona física o jurídica, autoridad pública, servicio o cualquier otro organismo que, sólo o conjuntamente con otros, trate datos personales por cuenta del responsable del tratamiento.

h) Consentimiento del interesado: toda manifestación de voluntad, libre, inequívoca, específica e informada, mediante la que el interesado consienta el tratamiento de datos personales que le conciernen.

i) Cesión o comunicación de datos: toda revelación de datos realizada a una persona distinta del interesado.

j) Fuentes accesibles al público: aquellos ficheros cuya consulta puede ser realizada, por cualquier persona, no impedida por una norma limitativa o sin más exigen- 
cia que, en su caso, el abono de una contraprestación. Tienen la consideración de fuentes de acceso público, exclusivamente, el censo promocional, los repertorios telefónicos en los términos previstos por su normativa específica y las listas de personas pertenecientes a grupos de profesionales que contengan únicamente los datos de nombre, título, profesión, actividad, grado académico, dirección e indicación de su pertenencia al grupo. Asimismo, tienen el carácter de fuentes de acceso público, los diarios y boletines oficiales y los medios de comunicación.

\section{TÍTULO II}

\section{Principios de la protección de datos}

\section{Artículo 4. Calidad de los datos}

1. Los datos de carácter personal sólo se podrán recoger para su tratamiento, así como someterlos a dicho tratamiento, cuando sean adecuados, pertinentes y no excesivos en relación con el ámbito y las finalidades determinadas, explícitas y legítimas para las que se hayan obtenido.

2. Los datos de carácter personal objeto de tratamiento no podrán usarse para finalidades incompatibles con aquellas para las que los datos hubieran sido recogidos. No se considerará incompatible el tratamiento posterior de éstos con fines históricos, estadísticos o científicos.

3. Los datos de carácter personal serán exactos y puestos al día de forma que respondan con veracidad a la situación actual del afectado.

4. Si los datos de carácter personal registrados resultaran ser inexactos, en todo o en parte, o incompletos, serán cancelados y sustituidos de oficio por los correspondientes datos rectificados 0 completados, sin perjuicio de las facultades que a los afectados reconoce el artículo 16 .

5. Los datos de carácter personal serán cancelados cuando hayan dejado de ser necesarios o pertinentes para la finalidad para la cual hubieran sido recabados o registrados.

No serán conservados en forma que permita la identificación del interesado durante un período superior al necesario para los fines en base a los cuales hubieran sido recabados o registrados.

Reglamentariamente se determinará el procedimiento por el que, por excepción, atendidos los valores históricos, estadísticos o científicos de acuerdo con la legislación específica, se decida el mantenimiento íntegro de determinados datos.

6. Los datos de carácter personal serán almacenados de forma que permitan el ejercicio del derecho de acceso, salvo que sean legalmente cancelados.

7. Se prohíbe la recogida de datos por medios fraudulentos, desleales o ilícitos.

\section{Artículo 5. Derecho de información en la recogida de datos}

1. Los interesados a los que se soliciten datos personales deberán ser previamente informados de modo expreso, preciso e inequívoco:

a) De la existencia de un fichero o tratamiento de datos de carácter personal, de la finalidad de la recogida de éstos y de los destinatarios de la información.

b) Del carácter obligatorio o facultativo de su respuesta a las preguntas que les sean planteadas.

c) De las consecuencias de la obtención de los datos o de la negativa a suministrarlos.

d) De la posibilidad de ejercitar los derechos de acceso, rectificación, cancelación y oposición.

e) De la identidad y dirección del responsable del tratamiento o, en su caso, de su representante.

Cuando el responsable del tratamiento no esté establecido en el territorio de la Unión Europea y utilice en el tratamiento de datos medios situados en territorio español, deberá designar, salvo que tales medios se utilicen con fines de trámite, un representante en España, sin perjuicio de las acciones que pudieran emprenderse contra el propio responsable del tratamiento.

2. Cuando se utilicen cuestionarios $u$ otros impresos para la recogida, figurarán en los mismos, en forma claramente legible, las advertencias a que se refiere el apartado anterior. 
3. No será necesaria la información a que se refieren las letras b), c) y d) del apartado 1 si el contenido de ella se deduce claramente de la naturaleza de los datos personales que se solicitan o de las circunstancias en que se recaban.

5. No será de aplicación lo dispuesto en el apartado anterior cuando expresamente una ley lo prevea, cuando el tratamiento tenga fines históricos, estadísticos o científicos, o cuando la información al interesado resulte imposible o exija esfuerzos desproporcionados, a criterio de la Agencia de Protección de Datos o del organismo autonómico equivalente, en consideración al número de interesados, a la antigüedad de los datos y a las posibles medidas compensatorias.

\section{Artículo 6. Consentimiento del afectado}

2. No será preciso el consentimiento..., cuando el tratamiento de los datos tenga por finalidad proteger un interés vital del interesado en los términos del artículo 7, apartado 6, de la presente Ley.

4. En los casos en los que no sea necesario el consentimiento del afectado para el tratamiento de los datos de carácter personal, y siempre que una ley no disponga lo contrario, éste podrá oponerse a su tratamiento cuando existan motivos fundados y legítimos relativos a una concreta situación personal. En tal supuesto, el responsable del fichero excluirá del tratamiento los datos relativos al afectado.

Artículo 7. Datos especialmente protegidos

2. Sólo con el consentimiento expreso y por escrito del afectado podrán ser objeto de tratamiento los datos de carácter personal que revelen la ideología, afiliación sindical, religión y creencias...

3. Los datos de carácter personal que hagan referencia al origen racial, a la salud y a la vida sexual sólo podrán ser recabados, tratados y cedidos cuando, por razo- nes de interés general, así lo disponga una ley o el afectado consienta expresamente.

6 . No obstante lo dispuesto en los apartados anteriores, podrán ser objeto de tratamiento los datos de carácter personal a que se refieren los apartados 2 y 3 de este artículo, cuando dicho tratamiento resulte necesario para la prevención o para el diagnóstico médicos, la prestación de asistencia sanitaria o tratamientos médicos o la gestión de servicios sanitarios, siempre que dicho tratamiento de datos se realice por un profesional sanitario sujeto al secreto profesional o por otra persona sujeta asimismo a una obligación equivalente de secreto.

También podrán ser objeto de tratamiento los datos a que se refiere el párrafo anterior cuando el tratamiento sea necesario para salvaguardar el interés vital del afectado o de otra persona, en el supuesto de que el afectado esté física o jurídicamente incapacitado para dar su consentimiento.

\section{Artículo 8. Datos relativos a la salud}

Sin perjuicio de lo que se dispone en el artículo 11 respecto de la cesión, las instituciones y los centros sanitarios públicos y privados y los profesionales correspondientes podrán proceder al tratamiento de los datos de carácter personal relativos a la salud de las personas que a ellos acudan o hayan de ser tratados en los mismos, de acuerdo con lo dispuesto en la legislación estatal o autonómica sobre sanidad.

\section{Artículo 9. Seguridad de los datos}

1. El responsable del fichero, y, en su caso, el encargado del tratamiento deberán adoptar las medidas de índole técnica y organizativa necesarias que garanticen la seguridad de los datos de carácter personal y eviten su alteración, pérdida, tratamiento o acceso no autorizado, habida cuenta del estado de la tecnología, la naturaleza de los datos almacenados y los riesgos a que están expuestos, ya provengan de la acción humana o del medio físico o natural.

2. No se registrarán datos de carácter personal en ficheros que no reúnan las condiciones que se determinen por vía reglamentaria con respecto a su integridad 
y seguridad y a las de los centros de tratamiento, locales, equipos, sistemas y programas.

3. Reglamentariamente se establecerán los requisitos y condiciones que deban reunir los ficheros y las personas que intervengan en el tratamiento de los datos a que se refiere el artículo 7 de esta Ley.

\section{Artículo 10. Deber de secreto}

El responsable del fichero y quienes intervengan en cualquier fase del tratamiento de los datos de carácter personal están obligados al secreto profesional respecto de los mismos y al deber de guardarlos, obligaciones que subsistirán aun después de finalizar sus relaciones con el titular del fichero o, en su caso, con el responsable del mismo.

\section{Artículo 11. Comunicación de datos}

1. Los datos de carácter personal objeto del tratamiento sólo podrán ser comunicados a un tercero para el cumplimiento de fines directamente relacionados con las funciones legítimas del cedente y del cesionario con el previo consentimiento del interesado.

2. El consentimiento exigido en el apartado anterior no será preciso:

f) Cuando la cesión de datos de carácter personal relativos a la salud sea necesaria para solucionar una urgencia que requiera acceder a un fichero o para realizar los estudios epidemiológicos en los términos establecidos en la legislación sobre sanidad estatal o autonómica.

3. Será nulo el consentimiento para la comunicación de los datos de carácter personal a un tercero, cuando la información que se facilite al interesado no le permita conocer la finalidad a que destinarán los datos cuya comunicación se autoriza o el tipo de actividad de aquel a quien se pretenden comunicar.

4. El consentimiento para la comunicación de los datos de carácter personal tiene también un carácter de revocable.

5. Aquel a quien se comuniquen los datos de carácter personal se obliga, por el solo hecho de la comunicación, a la observancia de las disposiciones de la presente Ley.
6. Si la comunicación se efectúa previo procedimiento de disociación, no será aplicable lo establecido en los apartados anteriores.

\section{Artículo 12. Acceso a los datos por cuen- ta de terceros}

1. No se considerará comunicación de datos el acceso de un tercero a los datos cuando dicho acceso sea necesario para la prestación de un servicio al responsable del tratamiento.

2. La realización de tratamientos por cuenta de terceros deberá estar regulada en un contrato que deberá constar por escrito o en alguna otra forma que permita acreditar su celebración y contenido, estableciéndose expresamente que el encargado del tratamiento únicamente tratará los datos conforme a las instrucciones del responsable del tratamiento, que no los aplicará o utilizará con fin distinto al que figure en dicho contrato, ni los comunicará, ni siquiera para su conservación, a otras personas.

En el contrato se estipularán, asimismo, las medidas de seguridad a que se refiere el artículo 9 de esta Ley que el encargado del tratamiento está obligado a implementar.

3. Una vez cumplida la prestación contractual, los datos de carácter personal deberán ser destruidos o devueltos al responsable del tratamiento, al igual que cualquier soporte o documentos en que conste algún dato de carácter personal objeto del tratamiento.

4. En el caso de que el encargado del tratamiento destine los datos a otra finalidad, los comunique o los utilice incumpliendo las estipulaciones del contrato, será considerado también responsable del tratamiento, respondiendo de las infracciones en que hubiera incurrido personalmente.

\section{TÍTULO III \\ Derechos de las personas}

Artículo 14. Derecho de Consulta al Registro General de Protección de Datos

Cualquier persona podrá conocer, recabando a tal fin la información oportuna del 
Registro General de Protección de Datos, la existencia de tratamientos de datos de carácter personal, sus finalidades y la identidad del responsable del tratamiento. El Registro General será de consulta pública y gratuita.

\section{Artículo 15. Derecho de acceso}

1. El interesado tendrá derecho a solicitar y obtener gratuitamente información de sus datos de carácter personal sometidos a tratamiento, el origen de dichos datos, así como las comunicaciones realizadas o que se prevén hacer de los mismos.

2. La información podrá obtenerse mediante la mera consulta de los datos por medio de su visualización, o la indicación de los datos que son objeto de tratamiento mediante escrito, copia, telecopia o fotocopia, certificada o no, en forma legible e inteligible, sin utilizar claves o códigos que requieran el uso de dispositivos mecánicos específicos.

3 . El derecho de acceso a que se refiere este artículo sólo podrá ser ejercitado a intervalos no inferiores a doce meses, salvo que el interesado acredite un interés legítimo al efecto, en cuyo caso podrán ejercitarlo antes.

\section{Artículo 16. Derecho de rectificación y cancelación}

1. El responsable del tratamiento tendrá la obligación de hacer efectivo el derecho de rectificación o cancelación del interesado en el plazo de diez días.

2. Serán rectificados o cancelados, en su caso, los datos de carácter personal cuyo tratamiento no se ajuste a lo dispuesto en la presente Ley y, en particular, cuando tales datos resulten inexactos o incompletos.

3. La cancelación dará lugar al bloqueo de los datos, conservándose únicamente a disposición de las Administraciones públicas. Jueces y Tribunales, para la atención de las posibles responsabilidades nacidas del tratamiento, durante el plazo de prescripción de éstas. Cumplido el citado plazo deberá precederse a la supresión.

4. Si los datos rectificados o cancelados hubieran sido comunicados previamente, el responsable del tratamiento deberá notificar la rectificación o cancelación efectuada a quien se hayan comunicado, en el caso de que se mantenga el tratamiento por este último, que deberá también proceder a la cancelación.

5. Los datos de carácter personal deberán ser conservados durante los plazos previstos en las disposiciones aplicables o, en su caso, en las relaciones contractuales entre la persona o entidad responsable del tratamiento y el interesado.

Artículo 17. Procedimiento de oposición, acceso, rectificación o cancelación

1. Los procedimientos para ejercitar el derecho de oposición, acceso, así como los de rectificación y cancelación serán establecidos reglamentariamente.

2. No se exigirá contraprestación alguna por el ejercicio de los derechos de oposición, acceso, rectificación o cancelación.

\section{Artículo 18. Tutela de los derechos}

1. Las actuaciones contrarias a lo dispuesto en la presente Ley pueden ser objeto de reclamación por los interesados ante la Agencia de Protección de Datos, en la forma que reglamentariamente se determine.

2. El interesado al que se deniegue, total o parcialmente, el ejercicio de los derechos de oposición, acceso, rectificación o cancelación, podrá ponerlo en conocimiento de la Agencia de Protección de Datos o, en su caso, del organismo competente de cada Comunidad Autónoma, que deberá asegurarse de la procedencia o improcedencia de la denegación.

3. El plazo máximo en que debe dictarse la resolución expresa de tutela de derechos será de seis meses.

4. Contra las resoluciones de la Agencia de Protección de Datos procederá recurso contencioso-administrativo.

\section{Artículo 19. Derecho a indemnización}

1. Los interesados que, como consecuencia del incumplimiento de lo dispuesto en la presente Ley por el responsable o el encargado del tratamiento, sufran daño o lesión en sus bienes o derechos tendrán derecho a ser indemnizados.

2. Cuando se trate de ficheros de titularidad pública, la responsabilidad se exigirá 
de acuerdo con la legislación reguladora del régimen de responsabilidad de las Administraciones públicas.

3. En el caso de los ficheros de titularidad privada, la acción se ejercitará ante los órganos de la jurisdicción ordinaria.

\section{TÍTULO IV}

Disposiciones sectoriales

CAPÍTULO I

Ficheros de titularidad pública

$$
\text { CAPÍTULO II }
$$

Ficheros de titularidad privada

\section{TÍTULO V \\ Movimiento internacional de datos \\ TÍTULO VI \\ Agencia de Protección de Datos}

\author{
TÍTULO VII \\ Infracciones y sanciones
}

\section{Ley 16/2003, de 28 de mayo, de cohe- sión y calidad del Sistema Nacional de Salud.}

http://noticias.juridicas.com/base_datos/ Admin/l16-2003.html (Visitada el 27/11/2006)

Ley que pretende mejorar la calidad de la asistencia en el Sistema Nacional de Salud, garantizar la equidad y potenciar la participación de la sociedad.

Dada su extensión, incluyo el índice, el objeto y la declaración de principios.

\section{Artículo 1. Objeto}

El objeto de esta Ley es establecer el marco legal para las acciones de coordinación y cooperación de las Administraciones públicas sanitarias, en el ejercicio de sus respectivas competencias, de modo que se garantice la equidad, la calidad y la participación social en el Sistema Nacional de Salud, así como la colaboración activa de éste en la reducción de las desigualdades en salud.

Lo dispuesto en esta Ley será de aplicación a los servicios sanitarios de financiación pública y a los privados en los tér- minos previstos en el artículo 6 y en las disposiciones adicionales tercera y cuarta.

\section{Artículo 2. Principios generales}

Son principios que informan esta Ley:

a) La prestación de los servicios a los usuarios del Sistema Nacional de Salud en condiciones de igualdad efectiva y calidad.

b) El aseguramiento universal y público por parte del Estado.

c) La coordinación y la cooperación de las Administraciones públicas sanitarias para la superación de las desigualdades en salud.

d) La prestación de una atención integral a la salud, comprensiva tanto de su promoción como de la prevención de enfermedades, de la asistencia y de la rehabilitación, procurando un alto nivel de calidad.

e) La financiación pública del Sistema Nacional de Salud, de acuerdo con el vigente sistema de financiación autonómica.

f) La igualdad de oportunidades y la libre circulación de los profesionales en el conjunto del Sistema Nacional de Salud.

g) La colaboración entre los servicios sanitarios públicos y privados en la prestación de servicios a los usuarios del Sistema Nacional de Salud.

h) La colaboración de las oficinas de farmacia con el Sistema Nacional de Salud en el desempeño de la prestación farmacéutica.

\section{Sumario}

- CAPÍTUlO PRELIMINAR. DISPOSICIONES GENERALES

o Artículo 1. Objeto.

o Artículo 2. Principios generales.

o Artículo 3. Titulares de los derechos.

o Artículo 4. Derechos de los ciudadanos en el conjunto del Sistema Nacional de Salud.

o Artículo 5. Ámbito de aplicación.

o Artículo 6. Acciones en relación con las entidades sanitarias no integradas en el Sistema Nacional de Salud.

- CAPÍtUlo I. DE LAS PRESTACIONES

O SECCIÓN I. ORDENACIÓN DE PRESTACIONES 
- Artículo 7. Catálogo de prestaciones del Sistema Nacional de Salud.

- Artículo 8. Cartera de servicios del Sistema Nacional de Salud.

- Artículo 9. Personal y centros autorizados.

- Artículo 10. Financiación.

- Artículo 11. Prestaciones de salud pública.

- Artículo 12. Prestación de atención primaria.

- Artículo 13. Prestación de atención especializada.

- Artículo 14. Prestación de atención sociosanitaria.

- Artículo 15. Prestación de atención de urgencia.

- Artículo 16. Prestación farmacéutica.

- Artículo 17. Prestación ortoprotésica.

- Artículo 18. Prestación de productos dietéticos.

- Artículo 19. Prestación de transporte sanitario.

o SECCIÓN II. DESARROLLO Y ACTUALIZACIÓN DE LA CARTERA DE SERVICIOS

- Artículo 20. Desarrollo de la cartera de servicios del Sistema Nacional de Salud.

- Artículo 21. Actualización de la cartera de servicios del Sistema Nacional de Salud.

- Artículo 22. Uso tutelado.

o SECCIÓN III. GARANTías DE LAS PRESTACIONES

- Artículo 23. Garantía de accesibilidad.

- Artículo 24. Garantías de movilidad.

- Artículo 25. Garantías de tiempo.

- Artículo 26. Garantías de información.

- Artículo 27. Garantías de seguridad.

- Artículo 28. Garantías de calidad y servicios de referencia.

- Artículo 29. Ámbito de las garantías de seguridad y calidad.

- CAPÍtUlo II. DE LA FARMACIA

O SECCIÓN I. ORGANIZACIÓN Y EJERCICIO DE LAS COMPETENCIAS DEL ESTADO EN MATERIA DE FARMACIA

- Artículo 30. Competencias de la Administración General del Estado en materia de farmacia.
- Artículo 31. Ejercicio de las competencias del Estado en materia de farmacia.

- Artículo 32. órganos de dirección, control y de asesoramiento técnicocientífico de la Agencia Española de Medicamentos y Productos Sanitarios.

o SECCIÓN II. COLABORACIÓN DE LAS OFICINAS DE FARMACIA

- Artículo 33. Colaboración de las oficinas de farmacia.

- CAPÍtUlO III. DE lOS PROFESIONALES

o SECCIÓN I. PLANIFICACIÓN Y FORMACIÓN DE RECURSOS HUMANOS DEL SISTEMA NACIONAL DE SALUD

- Artículo 34. Principios generales.

- Artículo 35. Comisión de Recursos Humanos.

- Artículo 36. Formación de pregrado.

- Artículo 37. Formación de postgrado.

- Artículo 38. Formación continuada.

- Artículo 39. Formación profesional.

o SECCIÓN II. DESARROLLO PROFESIONAL Y MODERNIZACIÓN DEL SISTEMA NACIONAL DE SALUD

- Artículo 40. Desarrollo profesional.

- Artículo 41. Carrera profesional.

- Artículo 42. Evaluación de competencias.

- Artículo 43. Movilidad de los profesionales.

- CAPÍTUlo IV. DE LA INVESTIGACIÓN

o SECCIÓN I. LA INVESTIGACIÓN EN SALUD

- Artículo 44. Principios.

- Artículo 45. Iniciativa sectorial de investigación en salud en el marco del Plan Nacional de Investigación Científica, Desarrollo e Innovación Tecnológica.

- Artículo 46. Cooperación entre los sectores público y privado.

- Artículo 47. Los derechos, la salud y la seguridad de los pacientes en la investigación.

o SECCIÓN II. INSTITUTO DE SALUD CARLOS III

- Artículo 48. Funciones del Instituto de Salud Carlos III.

- Artículo 49. Centros de investigación del Sistema Nacional de Salud.

- Artículo 50. Institutos de investigación. 
- Artículo 51. Redes de investigación cooperativa.

- Artículo 52. Apoyo a la investigación.

- CAPítulo V. DEL SISTEMA DE INFORMACIÓN SANITARIA

o SECCIÓN I. SISTEMA DE INFORMACIÓN SANITARIA

- Artículo 53. Sistema de información sanitaria del Sistema Nacional de Salud.

- Artículo 54. Red de comunicaciones del Sistema Nacional de Salud.

- Artículo 55. Estadísticas de interés general supracomunitario.

- Artículo 56. Intercambio de información en salud entre organismos, centros y servicios del Sistema Nacional de Salud.

o SECCIÓN II. TARJETA SANITARIA INDIVIDUAL

- Artículo 57. La tarjeta sanitaria individual.

o SECCIÓN III. INSTITUTO DE INFORMACIÓN SANITARIA

- Artículo 58. Instituto de Información Sanitaria.

- CAPítulo VI. DE LA CALIDAD

o SECCIÓN I. ACCIONES EN MATERIA DE CALIDAD

- Artículo 59. Infraestructura de la calidad.

- Artículo 60. Agencia de Calidad del Sistema Nacional de Salud.

- Artículo 61. Planes de calidad del Sistema Nacional de Salud.

- Artículo 62. Evaluación externa.

O SECCIÓN II. EL OBSERVATORIO DEL SISTEMA NACIONAL DE SALUD

- Artículo 63. Observatorio del Sistema Nacional de Salud.

- CAPítulo VII. DE LOS PLANES INTEGRALES

o Artículo 64. Planes integrales de salud.

- CAPítulo VIII. DE LA SAlUd PÚBlica

o Artículo 65. Actuaciones coordinadas en salud pública y en seguridad alimentaria.

o Artículo 66. La cooperación en salud pública.

- CAPítulo IX. DE LA PARTICIPACIÓN SOCIAL

o Artículo 67. Consejo de Participación Social del Sistema Nacional de Salud.

o Artículo 68. Redes de conocimiento.

- CAPÍTULO X. DEL CONSEJO INTERTERRITORIAL o Artículo 69. Objeto.

o Artículo 70. Composición.

o Artículo 71. Funciones.

o Artículo 72. Acciones sanitarias conjuntas.

o Artículo 73. Régimen de Funcionamiento. Acuerdos.

o Artículo 74. Comisiones y grupos de trabajo.

o Artículo 75. Adscripción de organismos y estructuras de apoyo y cooperación al Consejo.

- CAPítUlO XI. DE LA ALTA INSPECCIÓN

o Artículo 76. Funciones y actividades de la Alta Inspección.

o Artículo 77. Plan de inspección sanitaria.

o Artículo 78. Memoria.

o Artículo 79. Coordinación y cooperación de la inspección en el Sistema Nacional de Salud.

- DisPosición ADICIONAl PRIMERA. Competencias del Estado en relación con Ceuta y Melilla.

- DISPOSICión AdICIONAl SEgunda. Asistencia sanitaria en el extranjero.

- DISPOSICIÓn ADICIONAL TERCERA. Competencias de otras Administraciones públicas en relación con las entidades sanitarias no integradas en el Sistema Nacional de Salud.

- DisPosición ADICIONAL CUARTA. Extensión del contenido de la cartera de servicios del Sistema Nacional de Salud.

- DisPosición adicional Quinta. Fondo de cohesión.

- DISPOSICIÓN ADICIONAL SEXTA. Transferencia a las comunidades autónomas de los servicios e instituciones sanitarias dependientes de Instituciones Penitenciarias.

- DisPosición adicional SÉPtima. Cooperación al desarrollo sanitario.

- Disposición adicional octava. Centros de referencia.

- DisPosición ADICIONAL NOVENA. Régimen económico y fiscal de Canarias.

- DISPOSICIÓN TRANSITORIA úNICA. Cartera de servicios.

- DISPOSICIÓN DEROGATORIA PRIMERA.

- DISPOSICIÓN DEROGATORIA SEGUNDA.

- DISPOSICIÓN FINAL PRIMERA. Título competencial.

- DisPosición FINAL SEgUNDA. Equilibrio financiero del Sistema Nacional de Salud. 
- DISPOSICIÓN FINAL TERCERA. Modificación de la Ley 25/1990, de 20 de diciembre, del Medicamento.

- DISPOSICIÓn FINAL CUARTA. Adaptación de la estructura orgánica del Ministerio de Sanidad y Consumo.
- DISPOSICIÓN FINAL QUINTA. Desarrollo normativo.

- DISPOSICIÓN FINAL SEXTA. Entrada en vigor. 